\title{
Matching Preclusion and Conditional Matching Preclusion for Bipartite Interconnection Networks I: Sufficient Conditions
}

\author{
Eddie Cheng \\ Department of Mathematics and Statistics, Oakland University, Rochester, Michigan 48309
}

Philip Hu

Yale University, New Haven, Connecticut 06511

Roger Jia

University of Michigan, Ann Arbor, Michigan 48109

\author{
László Lipták \\ Department of Mathematics and Statistics, Oakland University, Rochester, Michigan 48309
}

\begin{abstract}
The matching preclusion number of a graph is the minimum number of edges whose deletion results in a graph that has neither perfect matchings nor almost-perfect matchings. For many interconnection networks, the optimal sets are precisely those induced by a single vertex. Recently, the conditional matching preclusion number of a graph was introduced to look for obstruction sets beyond those induced by a single vertex. This number is defined to be the minimum number of edges whose deletion results in a graph with no isolated vertices that has neither perfect matchings nor almost-perfect matchings. In this article, we prove general results regarding the matching preclusion number and the conditional matching preclusion number as well as the classification of their respective optimal sets for bipartite graphs. () 2011 Wiley Periodicals, Inc. NETWORKS, Vol. 59(4), 349-356 2012
\end{abstract}

Keywords: interconnection networks; perfect matching; bipartite graphs

\section{INTRODUCTION}

A perfect matching in a graph is a set of edges such that every vertex is incident with exactly one edge in this set. An almost-perfect matching in a graph is a set of edges such that

Received September 2009; accepted February 2011

Correspondence to: L. Lipták; e-mail: liptak@oakland.edu

Contract grant sponsor: NSF-REU; Contract grant number: DMS 0649099 DOI 10.1002/net.20440

Published online 22 April 2011 in Wiley Online Library (wileyonlinelibrary.com).

(C) 2011 Wiley Periodicals, Inc. every vertex except one is incident with exactly one edge in this set, and the exceptional vertex is incident to none. So if a graph has a perfect matching, then it has an even number of vertices; if a graph has an almost-perfect matching, then it has an odd number of vertices. The matching preclusion number of a graph $G$, denoted by $\operatorname{mp}(G)$, is the minimum number of edges whose deletion leaves the resulting graph without a perfect matching or almost-perfect matching. Any such optimal set is called an optimal matching preclusion set. We define $\operatorname{mp}(G)=0$ if $G$ has neither a perfect matching nor an almost-perfect matching. This concept of matching preclusion was introduced by [5] and further studied by [6, 8]. They introduced this concept as a measure of robustness in the event of edge failure in interconnection networks, as well as a theoretical connection to conditional connectivity, "changing and unchanging of invariants" and extremal graph theory. We refer the readers to [5] for details and additional references.

Distributed processor architectures offer the advantage of improved connectivity and reliability. An important component of such a distributed system is the system topology, which defines the inter-processor communication architecture. In certain applications, every vertex requires a special partner at any given time, and the matching preclusion number measures the robustness of this requirement in the event of link failures as indicated in [5]. Hence in these interconnection networks, it is desirable to have the property that the only optimal matching preclusion sets are those whose elements are incident to a single vertex. 
Proposition 1.1. Let $G$ be a graph with an even number of vertices. Then $m p(G) \leq \delta(G)$, where $\delta(G)$ is the minimum degree of $G$.

Proof. Deleting all edges incident to a single vertex will give a graph with no perfect matchings and the result follows.

If $\operatorname{mp}(G)=\delta(G)$, then $G$ is maximally matched. We call an optimal solution of the form given in the proof of Proposition 1.1 a trivial optimal matching preclusion set. As mentioned earlier, it is desirable for an interconnection network to have only trivial optimal matching preclusion sets. A graph $G$ is super matched if $\operatorname{mp}(G)=\delta(G)$ and every optimal matching preclusion set is trivial. In a distributed system it is unlikely that, in the event of random link failures, all edges at some vertex fail. Hence it is natural to ask what the obstruction sets are for a graph with link failures to have a perfect matching subject to the condition that the faulty graph has no isolated vertices. This motivates the following definition [7]: The conditional matching preclusion number of a graph $G$, denoted by $\mathrm{mp}_{1}(G)$, is the minimum number of edges whose deletion leaves the resulting graph with no isolated vertices and without a perfect matching or almostperfect matching. Any such optimal set is called an optimal conditional matching preclusion set. We define $\mathrm{mp}_{1}(G)=0$ if $G$ has neither a perfect matching nor an almost-perfect matching. In this paper we will leave $\mathrm{mp}_{1}(G)$ undefined if a conditional matching preclusion set does not exist, that is, we cannot delete edges to satisfy both conditions in the definition.

If we delete edges so that the resulting graph has no isolated vertices, then a basic obstruction to a perfect matching will be the existence of a path $u-v-w$ in the resulting graph where the degree of $u$ and the degree of $w$ are 1 . So to produce such an obstruction set, one can pick any path $u-v-w$ in the original graph and delete all the edges incident to either $u$ or $w$ but not $v$. We define

$v_{e}(G)=\min \left\{d_{G}(u)+d_{G}(v)-2-y_{G}(u, v):\right.$

$u$ and $v$ are ends of a 2-path\},

where $d_{G}(u)$ is the degree of vertex $u$ and $y_{G}(u, v)=1$ if $u$ and $v$ are adjacent and 0 otherwise. (We will suppress $G$ and simply write $d$ and $y$ if it is clear from the context.) So mirroring Proposition 1.1, we have the following result. (We note that the condition $\delta(G) \geq 3$ ensures that the resulting graph has no isolated vertices after these edges have been deleted.)

Proposition 1.2. Let $G$ be a graph with an even number of vertices. Suppose every vertex in $G$ has degree at least 3. Then

$$
m p_{1}(G) \leq v_{e}(G) .
$$

If $\operatorname{mp}_{1}(G)=v_{e}(G)$, then $G$ is conditionally maximally matched. We call an optimal solution of the form induced by $v_{e}$ a trivial optimal conditional matching preclusion set. As mentioned earlier, the matching preclusion number measures the robustness of this requirement in the event of link failures, so it is desirable for an interconnection network to be super matched. Similarly, it is desirable to have the property that all optimal conditional matching preclusion sets are trivial as well. Such an interconnection network is conditionally super matched. Ref. [7] introduced this concept and considered the conditional matching preclusion problem for a number of basic networks including hypercubes, and it was proved that they have this desired property.

In addition to basic classes of graphs such as the complete graphs [5] and complete bipartite graphs [5], the matching preclusion problem was studied for many classes of popular interconnection networks, in particular, hypercubes [5], Cayley graphs generated by transposition trees (including the star graphs and bubble-sort graphs) [8], $(n, k)$-star graphs [8], arrangement graphs (generalizing both the star graphs and alternating group graphs) [6], $(n, k)$-bubble-sort graphs [9], Cayley graphs generated by 2-trees (a super class of the alternating group graphs) [6] and restricted HL-graphs [17]. These interconnection networks are super matched apart from small exceptional cases and boundary (though infinitely many) subclasses. Much less is known for the conditional matching preclusion problem. Apart from the complete graphs [7] and complete bipartite graphs [7], interesting classes including hypercubes [7] were studied. They are conditionally super matched apart from some exceptional cases. In addition, the conditional matching preclusion number is known for restricted HL-graphs [18] and bipartite HL-graphs [18].

The crux of most of the existing proofs involves Hamiltonicity results. This is a natural form of attack as a Hamiltonian cycle (on graphs with an even number of vertices) induces two edge-disjoint perfect matchings. Of course, the same can be said for the less stringent condition of a collection of even cycles that spans the graph. Nevertheless, Hamiltonicity results are used due to the fact that they are well studied and most of these interconnection networks have optimal Hamiltonian results. In particular, a typical result is if "many" vertices and/or edges are deleted, then the resulting graph has the property that there is a Hamiltonian path between every pair of vertices. (If the graph is bipartite, additional restrictions are needed.) These results are optimal in the sense that "many" refers to a number that is the best possible. See [12-15] for these types of Hamiltonian results for popular interconnection networks. Since Hamiltonicity results are stringent, it is more desirable to replace them with a less stringent condition in the sense that the verification of such a condition should not be NP-Complete. In this article, we provide such sufficient conditions for bipartite graphs to be super matched and conditionally super matched. These general results will be used to establish previously known super matched and conditionally super matched results for hypercubes as well as new results for hyper-stars and Cayley graphs generated by transposition trees (including the star graphs) in a companion paper (E. Cheng, P. Hu, R. Jia, and L. Lipták, Matching preclusion and conditional matching 
preclusion for bipartite interconnection networks II: Cayley graphs generated by transposition trees and hyperstars, Networks, submitted). Indeed, for the hyper-stars it is infeasible to use any Hamiltonicity result as it is an open problem whether they are Hamiltonian. (Regular hyper-stars are isomorphic to middle cubes, and it is a famous conjecture that middle cubes are Hamiltonian. This is often referred to as the revolving door conjecture.) This is one of our motivations to find general sufficient conditions.

We use standard graph theory terminology which can be found in West [20]. If the (vertex) connectivity of $G$ is $\delta(G)$, then $G$ is maximally connected; if the edge-connectivity of $G$ is $\delta(G)$, then $G$ is maximally edge-connected. A graph $G$ is (weakly) super connected if it is maximally connected and every disconnecting set of cardinality $\delta(G)$ is a set of neighbors of a vertex of minimum degree. Some authors use the term superconnected for this concept; it was introduced $[1-4,10]$ to understand more about the components that remain after a graph is disconnected. This concept can be strengthened. A graph $G$ is tightly super connected if, after the deletion of at most $\delta(G)$ vertices, the graph either remains connected or has exactly two connected components, one of which is a singleton. Some authors use the term vosperian property [11] or hyper-connectivity [16] for this concept. Note that a graph can be (weakly) super connected but not tightly super connected; for example, $K_{n, n}$. Naturally, these concepts can also apply to deletion of edges, but in this case, there is no difference between being weakly super edge-connected and being tightly super edge-connected. Therefore, we define a maximally edge-connected graph $G$ to be super edge-connected if the deletion of at most $\delta(G)$ edges results in either a connected graph or exactly two connected components, one of which is a singleton. (We note that unlike the deletion of vertices, here we can replace "deletion of at most $\delta(G)$ edges" by "deletion of $\delta(G)$ edges.")

This article is organized as follows: In Section 2, we give general results for the matching preclusion number and the classification of optimal solutions for bipartite graphs. In Section 3, we give general results for the conditional matching preclusion number and the classification of optimal solutions for bipartite graphs. Finally, we give some concluding remarks as well as an application of the general results developed here.

\section{MATCHING PRECLUSION FOR BIPARTITE GRAPHS}

In this section, we will establish a relationship between being super edge-connected and being super matched. In bipartite graphs, there is one obvious impediment to finding perfect matchings. Given a bipartite graph $G$ with bipartition $\left(V_{1}, V_{2}\right)$, consider a subset of vertices $W$ from one part. Let $N_{G}(W)$ be the set of vertices that are adjacent to a vertex in $W$. (We suppress $G$ if it is clear from the context.) If $\left|N_{G}(W)\right|<|W|$, then there is clearly no possibility of forming a perfect matching. Indeed, every matching would have to omit at least one vertex in $W$. Such a set $W$ is called an obstruction set. A well-known theorem regarding obstruction sets is Hall's Theorem:

Theorem 2.1 (Hall's Theorem). A bipartite graph $G$ has a perfect matching if and only if there are no obstruction sets in it.

An easy well-known corollary to Hall's Theorem is that the edges of a $k$-regular bipartite graph can be partitioned into $k$ perfect matchings. This fact immediately gives us the following result:

Theorem 2.2. Let $G$ be a $k$-regular bipartite graph. Then $G$ is maximally matched, that is, $\operatorname{mp}(G)=k$.

We now give a sufficient condition to guarantee that all optimal matching preclusion sets are trivial in regular bipartite graphs.

Theorem 2.3. Let $G$ be a k-regular bipartite graph that is super edge-connected. Then $G$ is super matched.

Proof. From Theorem 2.2, we know that $\operatorname{mp}(G)=k$. Now suppose that $G$ is not super matched. Then, by Hall's Theorem, after deleting some set of $k$ edges $F$ such that $G-F$ does not contain a singleton, there must exist an obstruction set $W$ in $G-F$.

Clearly the number of edges incident to vertices in $W$ must be less than or equal to the number of edges incident to vertices in $N_{G-F}(W)$. Since $G$ is $k$-regular, the number of edges incident to vertices in $W$ in the graph $G-F$ is at least $|W| k-k$. Similarly, the number of edges incident to vertices in $N_{G-F}(W)$ is at most $\left|N_{G-F}(W)\right| k$. Thus we have

$$
\left|N_{G-F}(W)\right| k \geq|W| k-k,
$$

so

$$
\left|N_{G-F}(W)\right| \geq|W|-1 \text {. }
$$

In fact, since $W$ is an obstruction set, we must have equality. Therefore the number of edges incident to vertices in $W$ is at least $|W| k-k$ while the number of edges incident to vertices in $N_{G-F}(W)$ is at most $(|W|-1) k=|W| k-k$. Then it must be the case that $N_{G-F}\left(N_{G-F}(W)\right)=W$. Hence there is no edge between $N_{G-F}(W) \cup W$ and $V(G)-\left(N_{G-F}(W) \cup W\right)$. So $G-F$ has at least two components with no singletons. This is impossible as $G$ is super edge-connected.

The natural question is to ask whether the condition in Theorem 2.3 is necessary. The answer is clearly no, as one can just take two copies of any super matched graphs. Then the resulting graph must be super matched but it is not even connected.

In the study of super connectedness, researchers usually deal with tightly super connectedness rather than super edgeconnectedness because for all practical purposes, the first one is a stronger requirement as the next theorem shows. 
Theorem 2.4. Let $\xi \geq 1$. Suppose a connected graph $G$ has the property that after deleting at most $m$ vertices, where $m \geq$ 1, G either remains connected or consists of two components, one of which has at most $\xi$ vertices. If $|V(G)| \geq m+2 \xi+2$, then after deleting at most $m$ edges, it is also the case that $G$ either remains connected or consists of two components, one of which has at most $\xi$ vertices.

Proof. Suppose by contradiction that this is not true for some graph $G$ with at least $m+2 \xi+2$ vertices. Then there exists a set $F$ of at most $m$ edges such that it is possible to allocate the vertices of $G$ into two sets, $C_{1}$ and $C_{2}$, each having at least $\xi+1$ elements, such that there are no edges incident to both a vertex in $C_{1}$ and a vertex in $C_{2}$ in $G-F$. Let $F_{c}$ be the set of edges in $G$ that are incident to both a vertex in $C_{1}$ and a vertex in $C_{2}$. Since $G$ is connected, we know that $F_{c} \neq \emptyset$. In addition, $F_{c} \subseteq F$, so $\left|F_{c}\right| \leq m$.

We will construct a set $V_{c}$ of vertices in $G$ such that the vertices in $G-V_{c}$ can be allocated into two sets $C_{a}$ and $C_{b}$, each having at least $\xi+1$ elements, such that there is no edge between $C_{a}$ and $C_{b}$ in $G-V_{c}$.

Label the elements of $F_{c}$ as $f_{1}, f_{2}, \ldots, f_{r}$, where $r \leq m$. Each $f_{i}, 1 \leq i \leq r$, has one endpoint in $C_{1}$ and one endpoint in $C_{2}$. Thus let $f_{i}=\left(u_{i}, v_{i}\right)$ such that $u_{i} \in C_{1}$ and $v_{i} \in C_{2}$. We construct $V_{c}$ as follows: Set $V_{c}:=\emptyset$. For $i=1$ to $m$, if $\left|C_{1}-V_{c}\right| \geq\left|C_{2}-V_{c}\right|$, then add $u_{i}$ to $V_{c}$ ( $u_{i}$ may already be in $V_{c}$ ), otherwise add $v_{i}$ to $V_{c}\left(v_{i}\right.$ may already be in $\left.V_{c}\right)$. We note that at the beginning of stage $i,\left|C_{1}-V_{c}\right|+\left|C_{2}-V_{c}\right| \geq$ $m+2 \xi+2-i+1=m+2 \xi+3-i$. Moreover, at the end of stage $i,\left|C_{1}-V_{c}\right|,\left|C_{2}-V_{c}\right| \geq \xi+\frac{m+3-i}{2}-1$. Hence at the end of the construction we have $\left|C_{1}-V_{c}\right|,\left|C_{2}-V_{c}\right| \geq \xi+\frac{3}{2}-1$. But these numbers are integers, so $\left|C_{1}-V_{c}\right|,\left|C_{2}-V_{c}\right| \geq \xi+1$. By construction, $\left|V_{c}\right| \leq m$, and there are no edges between vertices in $C_{a}=C_{1}-V_{c}$ and $C_{b}=C_{2}-V_{c}$. If either of the graphs induced by $C_{a}$ and $C_{b}$ is not connected, then $G-V_{c}$ has at least three components, which is not possible. So they must be connected. But then $G-V_{c}$ has two components, both with at least $\xi+1$ vertices, obtaining a contradiction.

Corollary 2.5. Let $H$ be a tightly super connected graph. If $H$ has more than $\delta(H)+4$ vertices, then it is super edgeconnected.

Proof. Set $\xi=1$ in Theorem 2.4 .

We note that the condition on the number of vertices is needed, as the result is not true in general. For example, a 5cycle is tightly super connected but not super edge-connected. Theorem 2.4 will also be applied later for conditional matching preclusions. For now, we combine Theorem 2.4 with Theorem 2.3 to state the following theorem:

Theorem 2.6. Let $G$ be a k-regular bipartite graph that is tightly super connected. If $G$ has at least $k+4$ vertices, then $G$ is super matched. In particular, if $k \geq 4$, then a tightly super connected $k$-regular bipartite graph is super matched.
Many classes of interconnection networks are known to be tightly super connected. We will apply this theorem later to solve the matching preclusion problem for a number of classes of interconnection networks.

The matching preclusion number is defined only for graphs with a perfect matching or an almost perfect matching. Indeed, interconnection networks are almost always regular, and they usually satisfy this condition. However, it may be of interest to extend this definition to non-regular graphs that may not have a perfect matching or an almost perfect matching. We will now discuss this extension for bipartite graphs.

Let $G$ be a bipartite graph with bipartition $\left(V_{1}, V_{2}\right)$. Let $\Delta\left(V_{1}\right)=\max \left\{d(v): v \in V_{1}\right\}$ and let $\delta\left(V_{1}\right)=\min \{d(v)$ : $\left.v \in V_{1}\right\}$. Similarly, we can define $\Delta\left(V_{2}\right)$ and $\delta\left(V_{2}\right)$. It is clear that if $\left|V_{1}\right| \neq\left|V_{2}\right|$, no perfect matching is possible. In that case we are interested in matchings that saturate one side. Assume $\left|V_{1}\right| \geq\left|V_{2}\right|$. A set of edges $M$ is called a saturating matching if every vertex in $V_{2}$ is incident to exactly one element of $M$ and every vertex in $V_{1}$ is incident to at most one element of $M$. We may also say that $M$ saturates $V_{2}$. We define the matching preclusion number of $G, \operatorname{mp}_{s}(G)$, as the minimum number of edges that must be deleted such that no saturating matchings remain in the resulting graph. We note that if $G$ is regular, then this definition reduces to the original definition. Similarly to Proposition 1.1, the following observation is immediate:

Proposition 2.7. Let $G$ be a bipartite graph with bipartition $\left(V_{1}, V_{2}\right)$, and let $\left|V_{1}\right| \geq\left|V_{2}\right|$. Then $m_{s}(G) \leq \delta\left(V_{2}\right)$.

Proof. This is true since deleting all the edges incident to a vertex of minimum degree in $V_{2}$ guarantees that no saturating matchings remain.

We refer to any matching preclusion set given in the proof of Proposition 2.7 as a trivial matching preclusion set. In addition, a bipartite graph $G$ with bipartition $\left(V_{1}, V_{2}\right)$ and $\left|V_{1}\right| \geq\left|V_{2}\right|$ is called super matched if $\operatorname{mp}_{s}(G)=\delta\left(V_{2}\right)$ and the only minimum matching preclusion sets are the trivial matching preclusion sets. We are now ready for our result on matching preclusion sets.

Theorem 2.8. Let $G$ be a bipartite graph with bipartition $\left(V_{1}, V_{2}\right)$. If $\Delta\left(V_{1}\right) \leq \delta\left(V_{2}\right)$, then $m p_{s}(G)=\delta\left(V_{2}\right)$. If $\Delta\left(V_{1}\right)<\delta\left(V_{2}\right)$, then $G$ is super matched.

Proof. Note that since $\Delta\left(V_{1}\right) \leq \delta\left(V_{2}\right)$, we have $\left|V_{1}\right| \geq$ $\left|V_{2}\right|$. Thus we are interested in matchings that saturate the vertices in $V_{2}$.

First we will show that $\mathrm{mp}_{s}(G)=\delta\left(V_{2}\right)$. Suppose by contradiction that $M$ is a minimum matching preclusion set and $|M|<\delta\left(V_{2}\right)$. Let $W$ be an obstruction set in $G-M$.

Clearly, the number of edges incident in $G-M$ to vertices in $W$ is less than or equal to the number of edges incident to vertices in $N_{G-M}(W)$. We note that $W \subseteq V_{2}$, therefore the 
first number is at least $|W| \delta\left(V_{2}\right)-\delta\left(V_{2}\right)+1$ and the second number is at most $\left|N_{G-M}(W)\right| \Delta\left(V_{1}\right)$. Thus we have

$$
\left|N_{G-M}(W)\right| \Delta\left(V_{1}\right) \geq|W| \delta\left(V_{2}\right)-\delta\left(V_{2}\right)+1 .
$$

Hence

$$
\left|N_{G-M}(W)\right| \geq(|W|-1) \frac{\delta\left(V_{2}\right)}{\Delta\left(V_{1}\right)}+\frac{1}{\Delta\left(V_{1}\right)},
$$

therefore

$$
\left|N_{G-M}(W)\right|>|W|-1
$$

In addition, since $\left|N_{G-M}(W)\right|$ is an integer, it must be the case that $\left|N_{G-M}(W)\right| \geq|W|$. But this is a contradiction, since $W$ is an obstruction set.

Now suppose by contradiction that $G$ is not super matched and $\Delta\left(V_{1}\right)<\delta\left(V_{2}\right)$. Let $F$ be a non-trivial minimum matching preclusion set of cardinality $\delta\left(V_{2}\right)$, and let $W$ be an obstruction set in $G-F$ such that $|W| \neq 1$. We know such an obstruction set exists, because $F$ is not a trivial matching preclusion set. Again, we have

$$
\left|N_{G-F}(W)\right| \Delta\left(V_{1}\right) \geq|W| \delta\left(V_{2}\right)-\delta\left(V_{2}\right) .
$$

Therefore

$$
\left|N_{G-F}(W)\right| \geq(|W|-1) \frac{\delta\left(V_{2}\right)}{\Delta\left(V_{1}\right)} .
$$

This simplifies to

$$
\left|N_{G-F}(W)\right|>|W|-1,
$$

because $\delta\left(V_{2}\right)<\Delta\left(V_{1}\right)$ and because $|W|-1 \neq 0$. We again note the fact that $\left|N_{G-F}(W)\right|$ is an integer, so $\left|N_{G-F}(W)\right| \geq$ $|W|$. Hence we have a contradiction.

Note that Theorem 2.8 does not require the condition of being super edge-connected. Observe that if we replace $\Delta\left(V_{1}\right)<\delta\left(V_{2}\right)$ by $\Delta\left(V_{1}\right) \leq \delta\left(V_{2}\right)$, then the statement will include regular bipartite graphs, for which our previous theorem requires an additional condition. The proof of Theorem 2.8 breaks down if $\Delta\left(V_{1}\right)=\delta\left(V_{2}\right)$ as the inequality in (1) is not necessarily true.

\section{CONDITIONAL MATCHING PRECLUSION FOR REGULAR BIPARTITE GRAPHS}

If $G$ is a $k$-regular bipartite graph, then $v_{e}(G)=2 k-2$ and hence $\operatorname{mp}_{1}(G) \leq 2 k-2$ by Proposition 1.2. In this section, we give a sufficient condition for $\operatorname{mp}_{1}(G)=2 k-$ 2 and one for $G$ to be conditionally super matched. Recall that the sufficient condition for a $k$-regular bipartite graph $G$ to be super matched is for $G$ to be super edge-connected, that is, if whenever at most $k$ edges are deleted, then the resulting graph is either connected or it has two components, one of which is a singleton. Then it is perhaps reasonable to look for a condition in which when "many" edges are deleted, the resulting graph is either connected or it has one big component and a couple of small components. It turns out "many" should be $3 k-6$ for $\operatorname{mp}_{1}(G)=2 k-2$ and $3 k-4$ for $G$ to be conditionally super matched. A graph is super $m$-edge-connected of order $q$ if with at most $m$ edges deleted, the resulting graph is either connected or it has one big component and a number of small components with at most $q$ vertices in total. So a super edge-connected graph $G$ is super $\delta(G)$-edge-connected of order 1 .

Theorem 3.1. Let $G$ be a $k$-regular bipartite graph that is super $(3 k-6)$-edge-connected of order 2 . Then $G$ is conditionally maximally matched, that is, $m p_{1}(G)=2 k-2$.

Proof. Suppose by contradiction that $\operatorname{mp}_{1}(G)<2 k-$ 2. Then let $F$ be a conditional matching preclusion set of cardinality less than or equal to $2 k-3$, and let $W$ be an obstruction set in the graph $G-F$. Let $\left(V_{1}, V_{2}\right)$, where $\left|V_{1}\right|=$ $\left|V_{2}\right|$, be the bipartition of $G$, and without loss of generality, let $W \subseteq V_{1}$ so that $N_{G-F}(W) \subseteq V_{2}$. Note that $|W|>1$ as $F$ is a conditional matching preclusion set.

Since $W$ is an obstruction set, $\left|N_{G-F}(W)\right| \leq|W|-1$, so there are at most $|W| k-k$ edges incident to vertices in $N_{G-F}(W)$. In addition, there are at least $|W| k-(2 k-3)$ edges incident to vertices in $W$. Hence the number of edges between $N_{G-F}(W)$ and $V_{1}-W$ is at most $(|W| k-k)-$ $(|W| k-(2 k-3))=k-3$. By deleting an additional $k-3$ edges, we can disconnect $W \cup N_{G-F}(W)$ from the rest of $G$. Therefore, we have found an edge set $F^{\prime}$ of cardinality $3 k-6$ whose deletion gives a disconnected graph. Since $G$ is super $(3 k-6)$-edge-connected of order 2 , it must be the case that $G-F^{\prime}$ consists of either two components, one of which has no more than two vertices, or three components, two of which are singletons. Consider the following cases.

CASE 1. $G-F^{\prime}$ consists of two components, one of which is a singleton. If $W \cup N_{G-F}(W)$ is a singleton, then $N_{G-F}(W)$ is empty, implying that $W$ has no neighbors in $G-F^{\prime}$. This means that $W$ has no neighbors in $G-F$ as well, as $F^{\prime}-F$ only contains edges between $N_{G-F}(W)$ and $V_{1}-W$. However, that means that $W$ is an isolated singleton in $G-F$, contradicting the fact that $F$ is a conditional matching preclusion set. So $G-\left(W \cup N_{G-F}(W)\right)$ is a singleton. The singleton must belong to $V_{2}$ because $\left|N_{G-F}(W)\right|<|W|$ and $\left|V_{1}\right|=\left|V_{2}\right|$. However, that means that the singleton existed after the deletion of set $F$ since $F^{\prime}-F$ only contains edges between $N_{G-F}(W)$ and $V_{1}-$ $W$. Because $F$ is a conditional matching preclusion set, its deletion should not isolate a vertex, which is a contradiction.

CASE 2. $G-F^{\prime}$ consists of three components, two of which are singletons. As in the previous case, $W \cup N_{G-F}(W)$ cannot be a singleton. If $W \cup N_{G-F}(W)$ were the combination of the two singletons, $N_{G-F}(W)$ would be empty by definition. Then the obstruction set $W$ consists of two vertices, hence there are two singletons in $G-F$. Again, we have a contradiction because $F$ is a conditional matching preclusion set. 
If $W \cup N_{G-F}(W)$ were the nonsingleton component, then the two singletons must belong to $V_{2}-N_{G-F}(W)$ because $\left|N_{G-F}(W)\right|<|W|$ and $\left|V_{1}\right|=\left|V_{2}\right|$. As in Case $1, F^{\prime}-F$ only contains edges between $N_{G-F}(W)$ and $V_{1}-W$. Since the two singletons are in $V_{2}-N_{G-F}(W)$, it must be the case that they were isolated in $G-F$. Again, we have a contradiction.

CASE 3. $G-F^{\prime}$ consists of two components, one of which is an edge. If $W \cup N_{G-F}(W)$ were the edge component, then $|W|=\left|N_{G-F}(W)\right|$, since the two end-vertices of the edge cannot belong to the same vertex set of the bipartition. This contradicts the fact that $|W|$ is an obstruction set. Now suppose that $W \cup N_{G-F}(W)$ is the other component. Again, the two end-vertices of the edge-component cannot belong to the same vertex set of the bipartition. So $\left|V_{1}-W\right|=1$ and $\left|V_{2}-N_{G-F}(W)\right|=1$. Hence $|W|=\left|N_{G-F}(W)\right|$, which is a contradiction.

Theorem 3.2. Let $G$ be a k-regular bipartite graph with $m p_{1}(G)=2 k-2$. If $G$ is super $(3 k-4)$-edge-connected of order 3 , then it is conditionally super matched.

Proof. Suppose $G$ is a $k$-regular bipartite graph that is super $(3 k-4)$-edge-connected of order 3 . Now assume by contradiction that after deleting an edge set $F$ of cardinality $2 k-2$ we have an obstruction set $W$, and $F$ is not a trivial conditional matching preclusion set. Again, let $\left(V_{1}, V_{2}\right)$ be the bipartition of $G$ with $\left|V_{1}\right|=\left|V_{2}\right|$, and without loss of generality, let $W \subseteq V_{1}$, so $N_{G-F}(W) \subseteq V_{2}$.

As in the proof of Theorem 3.1, we see that there are at least $|W| k-2 k+2$ edges adjacent to vertices in $W$ and at most $\left|N_{G-F}(W)\right| k=|W| k-k$ edges adjacent to vertices in $N_{G-F}(W)$. Hence the number of edges between $N_{G-F}(W)$ and $V_{1}-W$ is at most $(|W| k-k)-(|W| k-(2 k-2))=$ $k-2$. Therefore, by deleting an additional $k-2$ edges, we can disconnect the graph. We have found an edge set $F^{\prime}$ of cardinality $3 k-4$ whose deletion disconnects the graph.

$G$ is super $(3 k-4)$-edge-connected of order 3, so we must have one large component of cardinality at least $|V(G)|-3$. From the proof of Theorem 3.1, we cannot have two components, one of which is a singleton or an edge, or three components, two of which are singletons. Hence it must be the case that we have a large component containing all the vertices except three. Since the graph is bipartite, there are no 3 -cycles in it. Therefore, we must delete at least $3 k-2$ edges to isolate three singletons and at least $3 k-3$ edges to isolate a singleton and an edge. Thus, after deleting $F^{\prime}$, the only possible case is that we have two components, one of which is a path of length 2 . Let the end-vertices in the path be $u$ and $w$, and let the middle vertex be $v$. We have two cases.

CASE 1. $W \cup N_{G-F}(W)=V\left(G-F^{\prime}\right)-\{u, v, w\}$. It must be the case that $V_{2}=N_{G-F}(W) \cup\{u, w\}$, since $\left|N_{G-F}(W)\right|<$ $|W|$. Thus $V-W=\{v\}$, so the $k-2$ edges in $F^{\prime}-F$ are all incident to $v$. Hence the only edges incident to $u$ and $w$ in $G-F$ are $(u, v)$ and $(v, w)$, respectively. However, this means that $F$ must have been a trivial conditional matching preclusion set, which is a contradiction.
CASE 2. $\quad W \cup N_{G-F}(W)=\{u, v, w\}$. Since $\left|N_{G-F}(W)\right|<$ $|W|$, it must be the case that $N_{G-F}(W)=\{v\}$ and $W=\{u, w\}$. Thus, the $k-2$ edges in $F^{\prime}-F$ are all incident to $v$. Again, this means that the only edges incident to $u$ and $w$ in $G-F$ are $(u, v)$ and $(v, w)$, respectively. Therefore, $F$ must have been a trivial matching preclusion set, a contradiction.

If we wonder whether the condition in Theorem 3.2 is necessary, the answer is no. There is a slack in the proof that will enable us to strengthen the result slightly. We defer the discussion to the Appendix, as Theorem 3.2 should be strong enough for most applications. As mentioned earlier and illustrated by Theorem 2.4 , the deletion of vertices is a stronger condition in general, so most research concentrates on the study of super $m$-connected graphs of order $q$ defined as follows: A graph is super $m$-connected of order $q$ if when at most $m$ vertices are deleted, the resulting graph is either connected or it has one big component and a number of small components with at most $q$ vertices in total. Thus it is worthwhile to state two corollaries of Theorem 3.1 and Theorem 3.2, respectively, so that the condition is for deletion of vertices. We need a reformulation of Theorem 2.4 to a slightly more general form:

Lemma 3.3. Suppose $m \geq 1$. Let $G$ be a connected graph with at least $m+2 \xi+2$ vertices. Suppose there exist at most $m$ edges whose deletion results in a graph with components that can be partitioned into two groups, each with at least $\xi+1$ vertices. Then there exist at most $m$ vertices whose deletion also results in a graph with components that can be partitioned into two groups, each with at least $\xi+1$ vertices.

Proof. Suppose there exists a set $F$ of at most $m$ edges such that the components of $G-F$ can be partitioned into two groups where each group has at least $\xi+1$ elements. Let $C_{1}$ and $C_{2}$ be the vertices in the two groups, respectively. Let $F_{c}$ be the set of edges in $G$ that are incident to both a vertex in $C_{1}$ and a vertex in $C_{2}$. Since $G$ is connected, we know $F_{c} \neq \varnothing$. In addition, $F_{c} \subseteq F$, so $\left|F_{c}\right| \leq m$.

We will construct a set $V_{c}$ of vertices in $G$ such that the vertices in $G-V_{c}$ can be allocated into two sets $C_{a}$ and $C_{b}$, each of which has at least $\xi+1$ elements, and there is no edge between $C_{a}$ and $C_{b}$ in $G-V_{c}$. Now proceed as in the proof of Theorem 2.4 to obtain $V_{c}$.

Theorem 3.4. Let $k \geq 2$ and $q \geq 1$. Let $G$ be a $k$-regular graph with at least $\max \{m+2 q+2,3 q+1\}$ vertices. If $G$ is super m-connected of order $q$, then it is super m-edgeconnected of order $q$.

Proof. Suppose not. Then we can delete $m$ edges from $G$ and the resulting graph will have components $Y_{1}, Y_{2}, \ldots, Y_{r}$ in nonincreasing order with respect to their cardinalities. So $Y_{2}, Y_{3}, \ldots, Y_{r}$ have collectively at least $q+1$ vertices. Clearly $\left|Y_{1}\right| \geq 2$ as $G$ has more than $m$ edges. If $\left|V_{1}\right| \in\{2,3, \ldots, q\}$, then we can group some remaining small $Y_{i}$ 's with $Y_{1}$ to form $S$ and the rest to form $T$. We can choose the groupings so that 
$S$ has at least $q+1$ vertices and at most $2 q$ vertices. So both $S$ and $T$ have at least $q+1$ vertices as $G$ has at least $3 q+1$ vertices. If $\left|Y_{1}\right| \geq q+1$, then $S$ consists of $Y_{1}$ and $T$ consists of the other components. So $T$ also has at least $q+1$ vertices by assumption. Now apply Lemma 3.3 with $\xi=q$ to obtain a contradiction.

Corollary 3.5. Let $k \geq 2$. Suppose $G$ is a $k$-regularbipartite graph with at least $\max \{3 k, 7\}$ vertices that is super $(3 k-$ $6)$-connected of order 2. Then $m p_{1}(G)=2 k-2$.

Proof. Apply Theorem 3.1 and Theorem 3.4 with $m=$ $3 k-6$ and $q=2$. Note that $\max \{m+2 q+2,3 q+1\}=$ $\max \{3 k, 7\}$.

Corollary 3.6. Let $k \geq 2$. Suppose $G$ be a $k$-regular bipartite graph and $m p_{1}(G)=2 k-2$. If $G$ has at least $3 k+4$ vertices and it is super $(3 k-4)$-connected of order 3 , then it is conditionally super matched.

Proof. Apply Theorem 3.2 and Theorem 3.4 with $m=$ $3 k-4$ and $q=3$. Note that $\max \{m+2 q+2,3 q+1\}=$ $\max \{3 k+4,10\}=3 k+4$ as $k \geq 2$.

\section{CONCLUSION AND AN APPLICATION}

In this article, we gave a comprehensive study of matching preclusion and conditional matching preclusion for bipartite interconnection networks. Earlier articles on this topics established such results by ad-hoc methods, and such methods usually involved results regarding fault Hamiltonicity. Moreover, there were no general sufficient conditions for a network to be maximally matched, super matched, conditionally maximally matched or conditionally super matched. Our goal for this article is three-fold. The first is to find a general sufficient condition for bipartite graphs. The second is to obtain a result with a condition that is not as stringent as results regarding fault Hamiltonicity. Finally, the verification of such a condition should be computationally efficient. Each of Theorem 2.2 (a well-known classical result), Theorem 2.3, Theorem 3.1, and Theorem 3.2 contains such a condition. We remark that the sufficient conditions given in this paper are in the spirit of a classical result of Plesník [19], which states that if $G$ is a $k$-regular $(k-1)$-edge-connected graph with an even number of vertices, then $G-F$ has a perfect matching for every $F \subseteq E$ with $|F| \leq k-1$. This immediately tells us that $\operatorname{mp}(G)=k$ for $k$-regular $(k-1)$-edge connected graphs with an even number of vertices.

Although the complete bipartite graphs are not suitable as interconnection networks, we will use them as an example to apply our results. Further applications to popular and suitable interconnection networks can be found in the companion paper (E. Cheng, P. Hu, R. Jia, and L. Lipták, Matching preclusion and conditional matching preclusion for bipartite interconnection networks II: Cayley graphs generated by transposition trees and hyperstars, Networks to appear).
Theorem 4.1 ([5]). Let $n \geq 2$. Then $m p\left(K_{n, n}\right)=n$, and $K_{n, n}$ is super matched.

Proof. Consider $K_{n, n}$ with bipartition $(A, B)$. Suppose that after $q$ edges are deleted, the graph is disconnected. We may assume that $G_{1}$ and $G_{2}$ are subgraphs of $K_{n, n}$ such that there are no edges between vertices of $G_{1}$ and $G_{2}$ in the resulting graph. Let $\left(A_{1}, B_{1}\right)$ be the bipartition of $G_{1}$ and $\left(A_{2}, B_{2}\right)$ be the bipartition of $G_{2}$ such that $A_{1} \cup A_{2}=A$ and $B_{1} \cup B_{2}=B$. Suppose $\left|A_{1}\right|=a_{1},\left|A_{2}\right|=a_{2},\left|B_{1}\right|=b_{1}$, $\left|B_{2}\right|=b_{2}$. Then we have deleted at least $a_{1} b_{2}+b_{1} a_{2}=$ $a_{1}\left(n-b_{1}\right)+b_{1}\left(n-a_{1}\right)=n\left(a_{1}+b_{1}\right)-2 a_{1} b_{1}$ edges. To prove Theorem 4.1, we use Theorem 2.3. So it is enough to show that $n\left(a_{1}+b_{1}\right)-2 a_{1} b_{1} \geq n+1$ subject to the condition that $2 \leq a_{1}+b_{1} \leq 2 n-2$. Observe that once $a_{1}+b_{1}$ is fixed, $n\left(a_{1}+b_{1}\right)-2 a_{1} b_{1}$ is minimized when $a_{1}=b_{1}$. So it suffices to show that $2 n x-2 x^{2} \geq n+1$ where $1 \leq x \leq n-1$. This is clearly true if $n \geq 3$. The case $n=2$ can be easily checked, and we are done.

Theorem 4.2 ([7]). Let $n \geq 3$. Then $m p_{1}\left(K_{n, n}\right)=2 n-2$, and $K_{n, n}$ is conditionally super matched.

Proof. We first use Theorem 3.1 for the first part and follow the same procedure. Hence it is enough to show that $n\left(a_{1}+b_{1}\right)-2 a_{1} b_{1} \geq 3 n-5$ subject to the condition that $3 \leq a_{1}+b_{1} \leq 2 n-3$. As before, it suffices to show that $2 n x-$ $2 x^{2} \geq 3 n-5$ where $3 / 2 \leq x \leq n-3 / 2$. This means it suffices to show that $2 n x-2 x^{2} \geq 3 n-5$ where $2 \leq x \leq n-2$. This is clearly true if $n \geq 3$. For the second part, we use Theorem 3.2 and follow the same procedure. Hence it is enough to show that $n\left(a_{1}+b_{1}\right)-2 a_{1} b_{1} \geq 3 n-3$ subject to the condition that $4 \leq a_{1}+b_{1} \leq 2 n-4$. Again, it suffices to show that $2 n x-2 x^{2} \geq 3 n-3$ where $2 \leq x \leq n-2$. This is clearly true if $n \geq 5$. The cases $n=3,4$ can be easily checked, and we are done.

\section{Acknowledgments}

We thank the anonymous referee for a number of helpful comments and suggestions including the recommendation that the manuscript be divided into two smaller articles.

\section{APPENDIX: STRENGTHENING OF THEOREM 3.2}

As mentioned after the proof of Theorem 3.2, the proof has a slack we can take advantage of. A bipartite graph is equitable if the two bipartition sets have the same cardinality. A bipartite graph is almost super $m$-edge-connected of order $q$ if when at most $m$ edges are deleted, the resulting graph satisfies one of the following: (1) it is connected, (2) it has one big component and a number of small components with at most $q$ vertices in total, and (3) every component is equitable.

If we relax the condition in Theorem 3.2 from super $(3 k-$ 4)-edge-connected of order 3 to almost super $(3 k-4)$-edgeconnected of order 3 , the result is still true. In fact, the proof is virtually the same. 
Theorem A.1. Let $G$ be a k-regular bipartite graph with $m p_{1}(G)=2 k-2$. If $G$ is almost super $(3 k-4)$-edgeconnected of order 3 , then it is conditionally super matched.

Proof. We proceed as in the proof of Theorem 3.2. Suppose $G$ is a $k$-regular bipartite graph that is almost super $(3 k-4)$-edge-connected of order 3 . Now assume that after deleting an edge set $F$ of cardinality $2 k-2$ we have an obstruction set $W$, and $F$ is not a trivial conditional matching preclusion set. Let $\left(V_{1}, V_{2}\right)$ be the bipartition of $G$ with $\left|V_{1}\right|=\left|V_{2}\right|$, and without loss of generality, let $W \subseteq V_{1}$, so $N_{G-F}(W) \subseteq V_{2}$. Note that $|W|>1$ as $F$ is a conditional matching preclusion set. Then following the same argument as in the proof of Theorem 3.2, we can delete up to $k-2$ additional edges to disconnect $W \cup N_{G-F}(W)$ from the rest of $G$. Therefore, we have found an edge set $F^{\prime}$ of cardinality $3 k-4$ whose deletion results in a disconnected graph. So $G-F^{\prime}$ is disconnected, and its components can be partitioned into two groups such that the first is the subgraph of $G-F^{\prime}$ induced by $W \cup N_{G-F}(W)$, and the second is the subgraph of $G-F^{\prime}$ induced by $\left(V_{1}-W\right) \cup\left(V_{2}-N_{G-F}(W)\right)$. Let $G_{1}$ be the first graph and $G_{2}$ be the second graph. Since $W$ is an obstruction set, $G_{1}$ is not equitable. Hence at least one component of $G-F^{\prime}$ is not equitable. But $G-F^{\prime}$ is almost super $(3 k-4)$-edge-connected of order 3 . Therefore $G-F^{\prime}$ has one big component plus a number of small components with at most three vertices in total. Now we can finish the proof as in the proof of Theorem 3.2.

\section{REFERENCES}

[1] C. Balbuena, K. Marshall, and L.P. Montejano, On the connectivity and superconnected graphs with small diameter, Discrete Appl Math 158 (2010), 397-403.

[2] D. Bauer, F. Boesch, C. Suffel, and R. Tindell, "Connectivity extremal problems and the design of reliable probabilistic networks," The Theory and Application of Graphs, Y. Alavi and G. Chartrand (Editors), Wiley, New York, 1981, pp. 89-98.

[3] F.T. Boesch, Synthesis of reliable networks - A survey, IEEE Trans Reliab 35 (1986), 240-246.

[4] F.T. Boesch and R. Tindell, Circulants and their connectivities, J Graph Theory 8 (1984), 487-499.
[5] R. Brigham, F. Harary, E. Violin, and J. Yellen, Perfect-matching preclusion, Congress Numer 174 (2005), 185-192.

[6] E. Cheng, L. Lesniak, M. Lipman, and L. Lipták, Matching preclusion for alternating group graphs and their generalizations, Int J Found Comput Sci 19 (2008), 1413-1437.

[7] E. Cheng, L. Lesniak, M. Lipman, and L. Lipták, Conditional matching preclusion sets, Inf Sci 179 (2009), 1092-1101.

[8] E. Cheng and L. Lipták, Matching preclusion for some interconnection networks, Networks 50 (2007), 173-180.

[9] E. Cheng, L. Lipták, and D. Sherman, Matching preclusion for the $(n, k)$-bubble-sort graphs, Int J Comput Math 87 (2010), 2408-2418.

[10] M.A. Fiol, J. Fábrega, and M. Escudero, Short paths and connectivity in graphs and digraphs, Ars Combin 29B (1990), $17-31$.

[11] Y.O. Hamidoune, A.S. Lladó, and O. Serra, Vosperian and superconnected abelian Cayley digraphs, Graphs Combin 7 (1991), 143-152.

[12] S. Hsieh, G. Chen, and C. Ho, Fault-free hamiltonian cycles in faulty arrangement graphs, IEEE Trans Parallel Distrib Syst 10 (1999), 223-237.

[13] H. Hsu, Y. Hsieh, J. Tan, and L. Hsu, Fault Hamiltonicity and fault Hamiltonian connectivity of the $(n, k)$-star graphs, Networks 42 (2003), 189-201.

[14] H.C. Hsu, T.K. Li, J. Tan, and L.H. Hsu, Fault Hamiltonicity and fault Hamiltonian connectivity of the arrangement graphs, IEEE Trans Comput 53 (2004), 39-53.

[15] T.K. Li, J. Tan, and L.H. Hsu, Hyper Hamiltonian laceability on edge fault star graph, Inf Sci 165 (2004), 59-71.

[16] X. Liang, J. Meng, and Z. Zhang, Super-connectivity and hyper-connectivity of vertex transitive bipartite graphs, Graphs Combin 23 (2007), 309-314.

[17] J.H. Park, Matching preclusion problem in restricted HLgraphs and recursive circulant $g\left(2^{m}, 4\right)$, J KIISE 35 (2008), 60-65.

[18] J.H. Park and S. Son, Conditional matching preclusion for hypercube-like interconnection networks, Theoret Comput Sci 410 (2009), 27-29.

[19] J. Plesník, Connectivity of regular graphs and the existence of 1-factors, Matematický Časop 22 (1972), 310-318.

[20] D. West, Introduction to graph theory, Prentice Hall, Upper Saddle River, NY, 1996. 